\title{
Luis Enrique Alonso, Carlos J. Fernández Rodríguez y Rafael Ibáñez Rojo, R. (Eds.). Estudios sociales sobre el consumo. Madrid: Centro de investigaciones sociológicas, 2020
}

\author{
Carlos de Castro Pericacho \\ Universidad Autónoma de Madrid, España. \\ c.decastro@uam.es
}

Luis Enrique Alonso, una de las figuras más reconocidas de la sociología española desde la década de los ochenta, Carlos J. Fernández y Rafael Ibáñez constituyen un grupo de investigación en la Universidad Autónoma de Madrid que ha dedicado aproximadamente los últimos 15 años a realizar las que probablemente sean las investigaciones sobre consumo, trabajo y ciudadanía más relevantes en el campo de la sociología española. Sus numerosas publicaciones durante ese periodo dan cuenta de ello. Pero tan importante como su prolífica trayectoria, ha sido (y es) su contribución a la articulación de una comunidad de investigadores e investigadoras del campo de la sociología del consumo desde la dirección del Comité de Investigación de Sociología del Consumo de la FES. De hecho, este volumen puede considerarse como un estado de la cuestión del campo de la sociología del consumo en España. En él participan algunos de los investigadores más reconocidos de todas las franjas generacionales desde los más reputados y consolidados hasta los/as más jóvenes. Esta apertura en la selección de voces también se refleja en el propio contenido del volumen. Los compiladores sostienen que la pluralidad o fragmentación de un objeto de investigación como el consumo en las sociedades contemporáneas requiere una aproximación plural desde el punto de vista epistemológico y metodológico. Así pues, el volumen recoge una amplia muestra de los paradigmas teóricos presentes en la sociología del consumo, así como de las aproximaciones metodológicas.

En coherencia con ello, el volumen se divide en cuatro bloques. En el primero de ellos, aborda el proceso de configuración histórica de la sociedad de consumo en España, así como de su constitución como objeto de estudio sociológico y de la gestación de las herramientas metodológicas (especialmente el grupo de discusión) para ello. En su capítulo Rodríguez Victoriano describe una de las principales singularidades de la formación de la sociedad de consumo en España desde finales de los 50: la contradicción entre, por un lado, una austeridad de una generación socializada en la pobreza y en la escasez de la posguerra y sometida a los valores sacrificiales del nacionalcatolicismo $y$, por otro lado, los intereses del capital nacional por integrar la economía española en los circuitos internacionales desplegando una norma de consumo similar a la europea. Los planes de estabilización de 1959 impulsados por los tecnócratas del Opus Dei desencadenaron los procesos (industrialización, aumentos salariales, apertura a mercados de bienes, etc....) que de manera abrupta insertaron a la sociedad española de manera muy desigual en algo equiparable al consumo de masas. 
La escuela del cualitativismo crítico liderada por Jesús Ibañez, Alfonso Ortí y Angel de Lucas se constituyó, entre otras cosas descritas en el fascinante artículo de Rodríguez Victoriano, en torno a la identificación de esa contradicción. De hecho, fue este grupo de intelectuales quienes empezaron a constituir el propio campo de la investigación sociológica del consumo en España en torno a esa contradicción. Expulsados de la academia por su militancia contra la dictadura franquista, desarrollaron su actividad en el ámbito de la empresa privada en la investigación de mercados. Inauguraron en España una forma de aproximarse al análisis del consumo que consideraba el consumo como una práctica que vinculaba a los individuos con las motivaciones y valores que subyacen a la estructura simbólica hegemónica de la sociedad. Consumir no era un acto individual que surgía de las preferencias individuales que se habrían gestado en el interior de la conciencia individual, sino que era y es una práctica colectiva. La investigación de este grupo se basó en un uso masivo de grupos de discusión ${ }^{1}$ con el fin de identificar esos discursos sociales que los actores generaban para apropiarse y dar sentido a los objetos que consumían. Rara vez eran la utilidad y la funcionalidad. Integración, pertenencia, expectativas de una vida mejor alejada de una penuria que en muchos casos fue vivida y que aún pesaba en el recuerdo, ambiciones de movilidad social ascendente, ... eran los motivos y valores que rebosaban simbólicamente los discursos. El capítulo de Gutiérrez Brito realiza, en este sentido, un análisis en profundidad de esta dimensión grupal y colectiva del consumo y de la importancia de adaptar el uso de los grupos de discusión para identificar las dinámicas de individualización del consumo en el marco de una evolución digital y fragmentaria de la demanda.

El capítulo de Brändle y Castillo ofrece una descripción minuciosa de la formación histórica de la sociedad del consumo en España en relación al desarrollo económico. El desarrollo del capitalismo de consumo dependía, por un lado, de una distribución de la masa salarial entre amplias capas de la población, algo que hacía depender la acumulación de capital no de los caprichos de los rentistas financieros, como denunciaba Keynes, sino de la sólida y ampliamente repartida base de los ingresos salariales. El mercado interior y, más concretamente, la capacidad de consumo de la clase trabajadora se constituía en el principal pilar de la acumulación. Pero, por otro lado, el desarrollo de este capitalismo de consumo dependía de la configuración de una industria que no sólo tuviera capacidad para abastecer las necesidades de consumo de la población, sino que también ofreciera ocupaciones con ingresos suficientes para alcanzar una capacidad de consumo suficiente. Brändle y Castillo muestran que estos dos requisitos (distribución de renta y sistema productivo) empiezan a cumplirse en las primeras décadas del siglo XX en algunos países europeos incluido España. En 1936 en España la producción industrial, el proceso de urbanización, el parque automovilístico... estaban en aumento, junto con la importancia de la economía de servicios, de la publicidad... Esa progresión quedó cancelada con el golpe de estado, la guerra civil y la posterior dictadura franquista. Los planes de estabilización de 1959 marcaron la apertura económica de la dictadura y empezaron a configurar la sociedad de consumo. Brändle y Castillo indican que la primera década de los sesenta estuvo marcada por cierto escepticismo y, sobre todo, por grandes desigualdades sociales, como muestran con el estudio sobre equipamiento doméstico en zonas industrializadas y zonas rurales realizado por José Castillo a mediados de los sesenta. La segunda década de los sesenta supuso una aceleración de la integración de la sociedad en los patrones de consumo y empezó a detectarse cómo la búsqueda de estatus y el sentimiento de privación ejercían una poderosa influencia en los hábitos de consumo de la emergente clase media. Los años setenta estabilizaron esa tendencia del modelo de consumo en un escenario de transformación política y cultural y de crisis económica. El desencanto con respecto a las enormes expectativas de cambio que había suscitado la transición a la democracia, la reconversión industrial de principios de los ochenta, la emergente precarización del empleo tras la primera reforma laboral de 1984, el crecimiento de los sectores culturales

1 Aunque Rodríguez Victoriano desvela cómo curiosamente fue el padre del cualitativismo, Jesús lbáñez, la persona que probablemente más encuestas precodificadas diseño y aplicó entre los años sesenta y setenta. 
y de servicios serían algunos de los factores que ayudarían a explicar el singular acceso de la sociedad española a un modelo de consumo marcado por un bienestar material y por una creciente segmentación y diferenciación.

Desde mediados de los ochenta, las pautas de consumo de la sociedad española son equiparables a las del resto de países europeos avanzados. Dentro de este marco, Fernando Conde analiza algunos cambios en las pautas de consumo a partir de los discursos producidos en un amplio trabajo de campo realizado en 2004, un momento de singular importancia puesto que son los años de aceleración y desenfreno consumista frenado casi de golpe por la crisis de 2008. Las pautas de consumo están marcadas tanto por las lógicas de consumo dominantes como por la dinámica de cambio en las formas de expresión cultural de las vinculaciones al consumo. Por un lado, presta atención a las lógicas sociales de consumo, esto es, a los procesos motivacionales y simbólicos que configuran los vínculos de los consumidores con los objetos de consumo. Distingue una lógica de la distinción (asociada a la posesión de un bien minoritario que goza de una elevada valoración social por su estatus social diferencial), una lógica de la diferencia (expresada en la imagen proyectada por el consumo de diferentes marcas, bienes, servicios asociados a un sistema de signos que vinculan a los consumidores con un grupo social u otro) y una lógica de la novedad (asociada con la diferenciación personal en el seno de los diversos grupos de pertenencia y a la búsqueda personal de innovación con la que se aspira a romper la rutina de lo conocido). Y, por otro lado, identifica tres niveles de profundidad en las formas de expresión cultural de las vinculaciones al consumo: natural, cultural y artificial. El nivel natural se refiere a aquello que se da por supuesto en la sociedad, a lo que pasa desapercibido por su aparente obviedad o por su familiaridad. Sería la forma de expresión cultural más generalizada por los grupos sociales. El nivel artificial se refiere a los elementos más ajenos a los hábitos, costumbres y gustos de la mayoría. Sería una forma minoritaria de la expresión cultural vinculada a los sectores más vanguardistas e innovadores. Por su parte, el nivel cultural se movería entre lo natural y lo artificial, entre la invisibilidad de lo rutinario y la vistosidad de la innovación. Sería una forma de expresión que empieza a consolidar en grupos cada vez más amplios alguna innovación. A partir de estas categorías, Conde trata de analizar cómo en cada momento histórico se produce una compleja articulación entre las lógicas sociales de consumo y las formas de expresión de los valores culturales asociados al consumo.

Desde una perspectiva y una aproximación muy diferentes, el capítulo de MartínLagos y Donat trata de auscultar la configuración a nivel europeo de un perfil de consumidor responsable a partir de la creación de un índice compuesto. El European Consumerisim Index $(\mathrm{ECl})$ ofrecería el retrato robot del consumidor ciudadano de la UE. Se trata de un indice que las autoras construyen a partir de una laboriosa tarea de recopilación y selección de numerosos indicadores vinculados explícita o implícitamente con el consumo elaborados por las instituciones de la Unión Europea desde 1975 hasta la actualidad. Su índice compuesto organiza los indicadores en tres dimensiones (conciencia ética y social, conciencia medioambiental y empoderamiento) y pretende medir el porcentaje de los consumidores que reflexionan sobre su proceso de compra incluyendo en esta reflexión la búsqueda de información sobre el proceso de elaboración del producto, la reivindicación de derechos y la preocupación por los efectos sociales y medioambientales de su acto de compra. A partir de este índice construyen un ranking europeo en el que los países del Norte y del Centro de Europa tienen un mayor porcentaje de consumidores empoderados, informados, conocedores de sus derechos, satisfechos con el funcionamiento del mercado y comprometidos con el medio ambiente que los países del Sur y Este de Europa. Se refleja así una mirada institucional de las diferencias entre los consumidores responsables de los países europeos.

El segundo bloque del volumen da cuenta de algunos de los principales paradigmas teóricos que orientan las investigaciones sociológicas sobre el consumo. El capítulo de los compiladores subraya la importancia de tomar en consideración la articulación entre las formas de producción y las formas de consumo para ofrecer una comprensión 
abarcadora de la complejidad de las prácticas de consumo. Encuentran en Bourdieu las herramientas para evitar algunos aspectos mecanicistas de la Escuela de la Regulación y la sociología marxista de los consumos colectivos. Desde la Escuela de la Regulación se enfatizaba la idea de que existe un tipo ideal de consumo para cada proceso de producción que está marcado por las características del modelo productivo, por las condiciones institucionales que regulan las relaciones entre capital y trabajo y por la estructura social que contribuye a generar. La estandarización del taylorismo y del fordismo traería consigo una norma social de consumo obrero, mientras que la fragmentación postfordista desplegaría una norma social de consumo más individualizado. Desde la sociología de los consumos colectivos, las prácticas de consumo se consideran como atravesadas por la contradicción entre las exigencias del beneficio y las necesidades de reproducción de la fuerza de trabajo. En este sentido, las prácticas de consumo se explicarían a partir de la socialización de las condiciones de reproducción de la fuerza de trabajo impulsada por el Estado. Sin negar esa interdependencia entre producción y consumo, los autores recurren a Bourdieu para evitar cierto determinismo y subrayar la autonomía de los sujetos en la configuración de las prácticas de consumo. Comprenden así las prácticas de consumo como comportamientos estructurados por la habitus de clase, aunque mediadas por el proceso de interiorización dirigido por el sujeto. Las prácticas de consumo están, por tanto, impregnadas de la construcción de sentido realizada por los sujetos en virtud de una combinación variable de capitales desde posiciones sociales que ofrecen unas limitadas posibilidades.

Moreno Pestaña también parte de Bourdieu para analizar el capital erótico en el trabajo. Considera el capital erótico como una especie de capital cultural que incluye procesos de valorización del cuerpo en varios niveles que definen los recursos eficientes en ciertos mercados de trabajo y entornos profesionales. Aborda el análisis del capital erótico a partir de la exclusión de los cuerpos que no encajan con los requisitos estéticos, a partir de las denuncias de la presión por ajustarse a un canon estético, y a partir de las prácticas de explotación de los cuerpos (p. 205). Por su parte, el capítulo de Barbeta revisita la clásica relación entre la sociología y psicoanálisis para explorar el carácter simbólico del consumo evitando algunos excesos y actualizándola para abordar la singularidad de las prácticas de consumo más individualizado en el contexto actual marcadas por un debilitamiento, que no desaparición, de los procesos motivacionales. Defiende un enfoque sociomotivacional del consumo en la medida en que lo socio motivacional respondería a un proceso concreto con el que los consumidores establecen un tipo de relación con respecto a un objeto. El proceso motivacional se situaría en la estructuración de concepciones y vivencias afectivas sobre determinados consumos, por lo que se ocuparía de analizar los procesos de fijación y religación. Las relaciones socio-motivacionales serían aquellas por las que un tipo de consumo responde a un fin construido socialmente por los consumidores desde contextos afectivos y socioestructurales específicos (p. 196).

En el análisis del consumo compulsivo en oposición al consumo reflexivo que realiza García resuenan algunos ecos de la aproximación psicoanalítica al consumo. García define el consumo compulsivo como conductas incontroladas, repetitivas, excesivas de adquisición de objetos y servicios que se daría como una respuesta a sentimientos negativos. El alivio de esos sentimientos sería una de las principales motivaciones de esa conducta que el sujeto no puede controlar (223). Presenta al consumidor compulsivo como la contraparte del modelo de consumidor que esbozan las teorías del consumidor reflexivo, y, a partir del ejemplo del aumento de los juegos de azar online, se pregunta por los factores sociales y culturales que pueden contribuir a la proliferación de la compulsividad en el consumo. El capítulo de Callejo vincula los modelos de consumo con los regímenes de acumulación de audiencia y centra su atención no tanto en los procesos de simbolización de las prácticas de consumo sino en un paso previo, en el modo en que el sistema de los medios de comunicación produce la atención que contribuye a constituir al propio consumidor. Toma, por tanto, la audiencia como un activo valorizable del capital. La audiencia sería un capital que se acumula para generar 
la plusvalía de la atención. Los regímenes de acumulación de audiencia representarían diversos modelos de consumidor según el modo en que se articule institucionalmente el sistema de comunicación que produce y se apropia de la atención (158-9). Callejo distingue entre regímenes de acumulación basados en la difusión (medios tradicionales) y regímenes basados en la lógica de la conexión otro relacional-reaccional (redes sociales). La transición entre uno y otro depende de su capacidad para producir atención de manera más extensa e intensa a menor coste.

Tras la indagación en los bloques anteriores en la constitución histórica de la sociedad de consumo y de algunas de las herramientas metodológicas y teóricas para investigarla, los tres siguientes bloques incluyen 16 capítulos que ofrecen un amplio abanico de análisis de corte más empírico sobre los cambios en las formas de consumo a raíz de la crisis económica y sobre la emergencia de prácticas de consumo vinculadas a los diversos ámbitos culturales y vinculadas a las plataformas digitales. En el corto espacio de una reseña es difícil describir el contenido de cada capítulo por lo que me dedicaré a mostrar de manera selectiva algunas de las áreas temáticas que abordan algunos de ellos.

El capítulo de Alonso, Fernández Rodríguez e Ibáñez Rojo aborda la profundización del consumo low cost en el despliegue de la economía de plataformas. Previamente a la crisis de 2008 , el segmento de consumo de bajo coste ya se había consolidado en parte como respuesta al deterioro de la capacidad adquisitiva de los trabajadores tras décadas de estancamiento salarial y desregulación laboral. La formación de un segmento de consumo de bajo coste era una adaptación de la oferta a las necesidades de reproducir la fuerza de trabajo de la manera más barata posible. La aparición de las plataformas digitales exacerba esa tendencia y establece una nueva vinculación entre las esferas del consumo y el trabajo. Las plataformas ofrecen la oportunidad de obtener un complemento a salarios reducidos: alquilando una habitación o la casa entera durante un periodo de tiempo o utilizar el vehículo particular para hacer repartos (Amazon, Glovo...) o para transportar a otras personas (Cabify, Blablacar...). Aunque el origen de las plataformas tenía una orientación colaborativa y solidaria, pronto se desplazó hacia una orientación descarnadamente mercantil (p. 247). En el capítulo se ofrecen algunas de las principales características de las nuevas formas de consumo de plataformas (sin distancias, sin intermediarios, abundancia gracias a una oferta ilimitada canalizada por internet o por aplicaciones, inmediatez gracias al sistema de reparto, ...) y algunas implicaciones sociales como la paradoja del acceso a servicios de bajo coste para mantener un estatus social que conduce a un deterioro de las condiciones de trabajo y de vida de la mayoría de la población.

El capítulo de Díaz de Rada presenta una clasificación de las comunidades autónomas según los patrones de gasto con el fin de explorar el impacto de la crisis en la orientación de los presupuestos en las diferentes comunidades autónomas. Divide a las comunidades en tres grupos según el nivel de ingresos y muestra cómo de manera general en casi todas las comunidades hay un aumento del peso de la enseñanza y de la vivienda y una disminución del peso de los alimentos y bebidas. Mientras en su capítulo, Díaz y García analizan los cambios en unos de los hábitos de consumo más importantes en nuestra sociedad: los hábitos alimentarios. Por un lado, muestra como la prolongación de los horarios de trabajo y de estudios contribuyen a configurar los peculiares horarios de las comidas en España: se retrasa el horario de las comidas y de las cenas. El capítulo muestra que este disciplinamiento horario de las comidas está vinculado con las formas familiares. Las familias con varios miembros adaptan sus horarios para intentar comer juntos. Las autoras muestran, por tanto, cómo la aparente irracionalidad de los horarios alimentarios en España esconde una racionalidad social que aspira a mantener el vinculo social entre los miembros familiares. Arribas y Fernández Trujillo analizan, por su parte, el papel del consumo en el proceso de integración de los inmigrantes latinoamericanos. Observan cómo el consumo de masas actúa como un potente factor que configura la cultura de consumo de los inmigrantes y que organiza su demanda. En el capítulo muestran cómo la oferta de una amplia y diversa gama de alimentos, prendas 
de vestir, teléfono móvil, equipamiento del hogar, vivienda o automóvil introduce a los inmigrantes en un estilo de vida que puede materializar las expectativas del proyecto migratorio. La crisis económica afectó especialmente a la población inmigrante y el artículo muestra cómo eso se dejó notar en su capacidad de consumo y en sus hábitos de consumo.

A la hora de abordar las relaciones entre consumo y prácticas culturales pueden destacarse las aportaciones que realizan una comparación transnacional de los patrones de consumo cultura con el fin de indagar en los rasgos de estratificación cultural en cada país (Katz-Gerro), la exhaustiva revisión del debate sobre el omnivorismo (Fernández Rodríguez y Heikkila), el análisis de la influencia del tipo de capital cultural generado en el entorno familiar y educativo sobre el consumo de una mayor diversidad de géneros artísticos y culturales (Herrera-Usagre) o una aproximación al consumo cultural desde una perspectiva relacional y pragmática que aspira a llevar el análisis de la democratización cultural hacia los contextos institucionales y relacionales que forjan la experiencia del gusto (Muntanyola-Saura). Por último, en el bloque que analiza los consumos digitales y los consumos colaborativos y sostenibles, nos encontramos con aportaciones que abordan el papel que juega la figura del community manager en la definición de las experiencias de consumo digital (Sádaba y Rendueles), que analizan el caso de Airbnb como emblema del consumo colaborativo mercantilizado (Gil García) o que tratan de situar en la agenda de los estudios sobre el consumo la cuestión de la sostenibilidad medioambiental de los hábitos de consumo, especialmente su conexión con las necesidades energéticas y materiales que requiere los productos de consumo (Álvarez y di Donato).

En definitiva, se trata de un volumen que, sin lugar a dudas, se convertirá en una obra de referencia indispensable tanto para expertos como para estudiantes interesados en los estudios sociales de consumo. 